Revue Française de Civilisation Britannique

XVII-2 | 2012

Minorités, intégration en Grande-Bretagne et dans les pays du Commonwealth

\title{
Doublement minoritaire : le cas de Matthew Mead, « independent minister » (c. 1630-1699)
}

Doubly in the Minority: the Case of Matthew Mead, "Independent Minister"

(c. 1630-1699)

\section{Arlette Sancery}

\section{OpenEdition}

Journals

Édition électronique

URL : http://journals.openedition.org/rfcb/667

DOI : $10.4000 /$ rfcb.667

ISSN : 2429-4373

Éditeur

CRECIB - Centre de recherche et d'études en civilisation britannique

Édition imprimée

Date de publication : 15 octobre 2012

Pagination : $19-24$

ISBN : 2-911580-37-0

ISSN : 0248-9015

Référence électronique

Arlette Sancery, «Doublement minoritaire : le cas de Matthew Mead, « independent minister »

(c. 1630-1699) », Revue Française de Civilisation Britannique [En ligne], XVII-2 | 2012, mis en ligne le 27 mars 2016, consulté le 22 avril 2019. URL : http://journals.openedition.org/rfcb/667 ; DOI : 10.4000/ rfcb.667

Ce document a été généré automatiquement le 22 avril 2019

\section{c)}

Revue française de civilisation britannique est mis à disposition selon les termes de la licence Creative Commons Attribution - Pas d'Utilisation Commerciale - Pas de Modification 4.0 International. 


\section{Doublement minoritaire : le cas de Matthew Mead, « independent minister » (c. 1630-1699)}

Doubly in the Minority: the Case of Matthew Mead, "Independent Minister" (c. 1630-1699)

\section{Arlette Sancery}

\section{NOTE DE L'AUTEUR}

« Independent » est, à l'époque, synonyme de « congregationalist ».

1 Le 6 février 1691, Matthew Mead prêchait à Stepney son célèbre sermon intitulé « Two sticks made one » d'après Ezéchiel 37,19 ${ }^{1}$. Le prophète évoluait bien sûr dans un contexte tragique, profondément différent de celui qui prévaut dans l'Angleterre du XVII siècle : le royaume du nord a été anéanti presque un siècle et demi auparavant, et la destruction de Jérusalem par les armées de Nabuchodonosor a eu lieu à deux reprises, en 598 puis en 587. Chaque fois, une grande partie des survivants a été déportée en Babylonie. La voix du prophète - qui a fait partie de la première vague de déportation - s'élève alors pour annoncer - « Oracle du Seigneur » - que les ossements desséchés seront revivifiés, que les deux morceaux de bois (sticks) séparés qui représentent les deux royaumes d'Israël et de Juda "seront un dans ma main", que l'unité - signe de la volonté de Dieu pour son peuple - reviendra, faisant la force du nouvel Israël. Pour Matthew Mead, la division de ceux qui refusent de faire allégeance à l'Église d'Angleterre et à ses évêques est une souffrance profonde. De plus, cette division entraîne une grande faiblesse face au roi. Donc il s'appuie sur les accents et l'ardeur du prophète pour prêcher en l'honneur de « the happy union", mouvement de réconciliation entre presbytériens et congrégationalistes lancé par John Howe ${ }^{2}$ l'année précédente. À peine quatre ans plus tard, tous les espoirs d'unité s'effondrent, au grand regret de Matthew Mead qui continuera inlassablement à plaider pour le rapprochement jusqu'à sa mort en 1699. 
2 Cet article n'est sans doute pas le lieu pour expliquer en détail les différences théologiques entre les deux mouvements, dont les presbytériens représentent une forte majorité, les «Independents » restant très minoritaires, même si les historiens n'avancent pas de chiffres précis. En réalité, l'opposition est moins doctrinale - les deux tendances restant proches du système calviniste - que pratique : leurs vues divergent sur le mode de fonctionnement des communautés. En quelques mots, rappelons que les Églises de la Réforme ne sont pas régies par un seul type d'organisation, car on ne peut tirer des Écritures un seul modèle organisationnel. Au contraire, l'Histoire montre que différents modèles peuvent exister et prétendre les uns et les autres à une légitimité scripturaire. On peut en dégager trois principaux :

1. le système hiérarchique ou épiscopal conservé par l'Église anglicane. Là, l'autorité vient d'en haut et se trouve déléguée vers la base. La structure hiérarchique est considérée comme d'inspiration divine. C'est elle qui assure l'unité de l'ensemble ;

2. le système congrégationaliste, où chaque assemblée est l'Église. Elle s'organise et se gouverne librement et noue des liens fraternels avec les autres assemblées. Une structure fédérative peut exister pour assurer des tâches communes, mais elle est dépourvue de toute autorité sur les églises locales, ne pouvant au mieux que formuler des recommandations ;

3. enfin, le régime presbytérien/synodal, où le mot « Église » recouvre à la fois l'église locale et l'union des églises locales. Le gouvernement de l'Église n'est pas confié aux seuls pasteurs ou ministres, mais à des «conseils presbytéraux » subordonnés aux synodes régionaux, euxmêmes dominés par le synode national. Aucune hiérarchie à caractère sacré dans ce système, mais un principe de collégialité et des mandats électifs, fondés sur la doctrine du sacerdoce universel.

3 C'est dans ce contexte, où le monde réformé cherche à tâtons une forme viable de fonctionnement, qu'évolue Matthew Mead.

Que savons-nous de ce Puritain indomptable, aussi connu en son temps que Richard Baxter ${ }^{3}$ mais aujourd'hui tombé dans l'oubli? Et surtout, comment expliquer l'impossible réconciliation de deux courants minoritaires sous le règne d'abord des Stuart, puis celui de William et Mary après la Glorieuse Révolution? C'est ce que nous allons tenter d'expliquer.

5 Le Oxford Dictionary of National Biography comporte dès 1894 un article fourni consacré à notre auteur et signé d'Alexander Gordon, lui-même connu pour ses études sur le puritanisme anglais ${ }^{4}$. Il nous apprend de nombreux détails sur Mead, dont nous nous contenterons aujourd'hui de résumer l'itinéraire exceptionnel. Son cheminement hors du commun révèle sa force de caractère et son esprit d'indépendance. Né vers 1630 dans le Derbyshire, il est admis à Cambridge (King's College) en 1649 mais refuse de signer «the Engagement $"^{5}$ et doit démissionner en 1651. Auparavant, il avait eu des controverses avec plusieurs de ses condisciples, et sa réputation de polémiste le précède à Londres où il va s'opposer à un autre grand dissenter ${ }^{6}$, William Greenhill qui loge au presbytère et occupe l'après-midi la chaire de Stepney où Mead prêche le matin. En 1658, il est nommé par Cromwell à la New Chapel de Shadwell (St Paul's), charge qu'il perd en 1660 avec la Restauration. Il obtient néanmoins un poste à la paroisse du St Sépulcre de Holborn, dont il est chassé en 1662 par l'Acte d'Uniformité 7 . Le Five Miles Act ${ }^{8}$ qui devient exécutoire en 1664 le conduit à s'exiler à Leyden, aux Pays-Bas, avant de retourner plus ou moins légalement à Londres où il semble avoir vécu la Grande Peste de 1665. En 1669, à Stepney, il devient l'assistant de Greenhill qu'il remplace à sa mort en 1671, devenant ainsi titulaire de la congrégation la plus importante de la capitale. Sa renommée de prédicateur 
est alors établie, comme le prouve la foule de ceux qui se pressent à ses prêches. Sa paroisse lui fait construire une grande maison à laquelle contribuent «les États de Hollande ", où il abritera James Peirce, the Exeter heretic, en 1680.

6 Sur la fin du règne de Charles II, les ennuis de Matthew Mead recommencent. Le roi est perçu comme trop proche de Louis XIV et soupçonné de volontés absolutistes. Son frère, le futur Jacques II, se convertit au catholicisme en 1673, ce qui exaspère une partie de la population, en particulier les républicains - qui vont par la suite former le parti Whig. Mead n'a pourtant aucun lien avec eux, mais fait l'objet d'une stricte surveillance policière comme tous les non-anglicans. Plusieurs fois interpellé par la police, il est finalement inculpé de complicité dans le complot de la Rye House ${ }^{9}$ qui va permettre au roi d'opérer un vaste coup de filet pour se débarrasser à la fois des opposants politiques et religieux. Arrêté et déféré devant le Privy Council en 1683, ses réponses sont si claires et son innocence si évidente que le roi lui-même requiert sa libération. En 1683, il succède à John Owen ${ }^{10}$ et prêche le mardi matin aux presbytériens et congrégationalistes réunis à la guilde des marchands de Pinners'Hall. La fougue avec laquelle il plaida un jour la cause de pasteurs réduits à la misère est restée célèbre : émues aux larmes, les dames présentes déposèrent dans la corbeille leurs bagues et leurs bijoux.

7 Après une nouvelle série de passages en Hollande, il revient en Angleterre en 1687 lorsque Jacques - devenu roi en 1683 - se déclare pour la liberté de conscience et s'oppose au Parlement qui tient à la suprématie de l'Église anglicane. Après la Glorieuse Révolution et l'avènement de William et Mary, Matthew Mead reprend son bâton de pèlerin et se consacre à sa dernière cause: la réconciliation des deux branches anglaises du protestantisme dissident, presbytériens et congrégationalistes. Déjà en 1683 il prêchait aux deux communautés réunies à Pinners'Hall, mais son sermon le plus célèbre reste celui de 1691 d'après Ezéchiel, dont la dédicace commence par ces mots: "The dissenting ministers of London, formerly called Congregationalists and Presbyterians, but now known by the name of United Brethren... ». Déçu par l'échec du rapprochement tant théologique que tactique qu'il souhaitait ardemment, il garde pourtant des amitiés dans les deux camps et poursuit une correspondance suivie avec leurs représentants dans les colonies américaines, en particulier avec la famille Mather. Sa quête obstinée d'union, malgré les obstacles innombrables, lui vaut le respect de tous et se traduit par les multiples témoignages de tristesse lors de sa mort en 1699. Cette brève biographie ne donne qu'une idée approximative du talent d'orateur de Matthew Mead, pour qui un sermon n'a pas seulement pour fonction d'expliquer l'Écriture - parfois ardue ou obscure pour le commun des mortels - et d'affermir ou propager la foi chrétienne. C'est aussi l'œuvre d'un artiste, d'un visionnaire, voire d'un prophète qui se veut, comme Baxter, la plume du Seigneur. Pourtant, malgré tout son talent et sa perspicacité, Mead échoue dans sa tentative de rapprochement. Comment pouvons-nous l'expliquer?

La situation politique est très complexe en cette fin du XVII ${ }^{\mathrm{e}}$ siècle anglais, tout autant que la situation religieuse d'ailleurs, et les historiens d'aujourd'hui expliquent qu'on ne peut séparer les deux domaines. Si aux yeux des Anglicans, les non-conformistes ne forment qu'une seule masse vague, le mouvement puritain depuis une trentaine d'années s'est subdivisé en plusieurs courants nés de profondes divisions doctrinales et/ou politiques, auxquels se surimposent des courants correspondants nés de l'émigration outre-Atlantique avec lesquels ils restent en contact. Les Puritains des débuts ne sont plus seuls. S'y sont ajoutés les Quakers, les Baptistes et de nombreuses autres sectes. Lors de la Restauration de 1660, nous avons vu que Charles II avait rétabli l'Église anglicane dans ses 
prérogatives d'avant la guerre civile (Acte d'Uniformité), ce qui marginalise les dissidents. Cependant, la plupart des prédicateurs interdits d'exercice continuent leur ministère " sous le manteau », malgré le Code de Clarendon ${ }^{11}$ complété par le Test Act ${ }^{12}$ qui tente de réduire au silence tous ces "schismatiques » et de les exclure de la fonction publique. Paradoxalement, ce sont les manœuvres de Jacques II pour imposer des nominations de catholiques à des postes de hauts fonctionnaires, dans l'Armée et à l'Université, qui vont rapprocher les évêques anglicans des non-conformistes et amener les plus intelligents d'entre ces derniers à comprendre la nécessité de l'unité, d'autant que la révocation de l'Édit de Nantes en 1685 et l'afflux de réfugiés huguenots en Angleterre montrent que le Protestantisme dans son ensemble est désormais menacé.

9 En effet, les archevêques et évêques anglicans eux-mêmes refusent en 1687 de lire la Déclaration d'Indulgence en chaire, et Baxter comme Mead s'associent à eux, déclinant de recevoir des mains d'un monarque ouvertement catholique des avantages et privilèges qui les auraient encore plus éloignés des autres protestants. Lorsque le roi fait emprisonner les sept évêques anglicans à la Tour de Londres, une délégation de dix pasteurs non-conformistes leur rend visite en prison, événement impensable quelques années plus tôt. Après l'acquittement des prélats, l'archevêque de Canterbury, Sancroft, écrit dans sa lettre pastorale qu'il souhaite désormais voir le clergé traiter «their dissenting brothers with great tenderness, to try to persuade them to conform, but in any case to make common cause with them for the defense of the Reformed faith $»^{13}$. Cet accès de bonne volonté ne va cependant pas durer très longtemps, car Guillaume d'Orange qui monte sur le trône après la chute de Jacques II est trop protestant, trop calviniste, aux yeux des grands prélats, si bien qu'une majorité de la Haute Église refuse le Comprehension Bill présenté à la Chambre des Lords en 1689. De même, l'énorme travail de la Jerusalem Chamber Commission, imprégné de l'esprit de conciliation mis en avant par le futur Archevêque de Canterbury, John Tillotson (de tendance latitudinaire), est refusé par les Communes comme par les Lords. Le Parlement est dissout en 1690 et tout espoir de voir les non-conformistes (dont le nombre est en forte augmentation, ce qui accroît encore les réticences des Anglicans) rejoindre l'Église d'Angleterre est aboli ${ }^{14}$.

Avant sa dissolution, le Parlement avait toutefois voté l'Acte de Tolérance, considéré par les parlementaires comme une concession amplement suffisante vis-à-vis des dissidents. Tolérés, mais non acceptés à la communion anglicane, les deux plus grands groupes dissidents commencent à envisager de s'unir, et traduisent cette idée dans les faits en constituant une banque de ressources financières destinée à la formation de leurs futurs pasteurs. La collecte des fonds est organisée par Matthew Mead pour les Congrégationalistes et John Howe, passé des Indépendants aux Presbytériens. Un Conseil d'administration réunit des membres des deux courants. John Howe propose alors que les ministres du culte émanant de cette formation commune prennent le nom de United Brethren, ce qui est accepté par environ cent membres du clergé à Londres. Ils signent une charte extrêmement importante, intitulée " The Heads of Agreement assented to by the United Ministers, in and about London, formerly called Presbyterian and Congregational». Ils y affirment qu'uniformité et unité sont deux notions différentes, que certains points de divergence théologique ne peuvent pas être résolus d'un seul coup, qu'il vaut mieux ne pas clore la discussion, qu'une structure paroissiale assez flexible est préférable à un cadre trop strict. La tendance générale est plutôt congrégationaliste, mais sans rigidité. À la signature de la Charte à Londres en avril 1691, Matthew Mead et Baxter laissent éclater 
leur joie, peu de temps avant la disparition de Baxter, qui meurt persuadé que la fédération est enfin une réalité, et une réalité durable.

11 Malheureusement, le successeur de Baxter à Pinners'Hall, Daniel Williams, est un polémiste qui accuse ses adversaires d'être des "Antinomiens", lui-même et ses partisans se faisant traiter d'« Arminiens » et même d'« Unitariens ». Les deux groupes se déchirent par des discussions théologiques effrénées, se séparent, et tout espoir d'unité s'évanouit quatre ans plus tard. Le fonds commun est dissout et si les Congrégationalistes continuent de se réunir à Pinners' Hall, les Presbytériens préfèrent désormais Salters' Hall. Les Anglicans en profitent pour renouveler leurs attaques contre les nonconformistes, ou ceux qui ne pratiquent qu'une conformité occasionnelle. La recherche d'une solution qui aurait profité à tous disparait devant la soif de pouvoir et l'individualisme des dirigeants. Être membre d'une minorité, nous le voyons, n'est en aucun cas une garantie de clairvoyance ou de bon sens. La division des deux branches est visiblement mortifère et les dissidents courent le risque tout simplement de disparaitre de la scène religieuse, privés de tous moyens de survivre. L'ironie de l'histoire réside dans le fait qu'aux États-Unis d'Amérique, le modèle de Charte (Heads of Agreement), rejeté en Angleterre, allait au contraire faire florès, peut-être d'ailleurs sous l'influence de Matthew Mead qui rédige une préface à la vie de Nathaniel Mather ${ }^{15}$, avec qui il avait entretenu une correspondance. Sans que Matthew Mead le sache, les dissenters allaient devoir attendre la mort de la reine Anne, leur farouche ennemie, et l'accession au trône d'Angleterre des premiers rois de Hanovre en 1714 pour obtenir enfin la tolérance.

\section{NOTES}

1. Toutes les citations de la Bible en français moderne sont tirées de la TOB (Traduction œcuménique de la Bible), éd. Le Cerf, Paris, 1972 pour le Nouveau Testament et 1975 pour l'Ancien Testament.

Pour ses propres citations, Matthew Mead n'utilise pas la Bible de 1611 (King James'Version), mais préfère la version anglaise de la Bible de Genève, dite Puritan Bible, London, 1588 "Imprinted in London by the deputies of Christopher Barker ".

2. John Howe (1630-1705) fut brièvement aumônier de Cromwell. Théologien et instigateur du mouvement presbytérien en Irlande, il fit partie de la délégation envoyée accueillir Guillaume d'Orange en 1688.

3. Richard Baxter (1615-1691), un des plus grands théologiens et prédicateurs protestants, respecté même de ses adversaires. Le juge Jeffreys s'acharne sur lui et l'envoie en prison à 70 ans. Des anglicans comme des puritains assisteront à ses funérailles.

4. Alexander GORDON, English Presbyterianism, The Christian Life, London, 1888.

5. «L'engagement » était celui d'utiliser le Book of Common Prayer (BCP) de règle à King's et refusé par les dissenters. Atténuées sous Cromwell (1599-1658), les pressions visant à rendre son usage obligatoire reprennent de plus belle à la Restauration.

6. Pour une définition plus fine du terme de " dissenter ", par opposition à "nonconformist », on peut se référer à l'ouvrage de Paul SANGSTER, History of the Free Churches, London, 1983. 
7. Act of Uniformity : CharlesII refuse la liberté de conscience aux non-conformistes. En 1661, le «Cavalier Parliament» propose de rendre obligatoire le BCP pour tous les Ministres du culte, proposition qui devient loi l'année suivante sous le nom d'Acte d'Uniformité. Les Puritains l'appellent "Bartholomew's Act », en référence à la St Barthélémy de sinistre mémoire, la loi devenant exécutoire le 24 août 1662. Elle fait obligation de réordonner tous les ministres du culte ne l'ayant pas été par un évêque et de « déjurer » (unswear) le serment prêté à la "Solemn League and Covenant" depuis 1642. En conséquence, $1 / 5^{\mathrm{e}}$ des membres du clergé puritain sont « éjectés » (the Great Ejectment), dont 1285 diplômés de l'Université, parmi lesquels de très grands noms.

8. Five Miles Act (Oxford Act ou Nonconformists Act) : passée sous Charles II en 1665 cette loi interdisait aux dissenting ministers de résider à moins de $8 \mathrm{~km}$ d'une paroisse d'où ils avaient été chassés. Ils ne pouvaient y retourner que s'ils adoptaient le BCP et promettaient de ne plus s'opposer au roi et à son gouvernement de l'Eglise et de l'Etat

9. Rye House plot (1683) : complot avorté contre le roi et son frère, qui donne lieu de la part des Tories à une répression sévère. Cela explique le mécontentement populaire qui s'exprime lors de la Glorious Revolution de 1688.

10. John Owen (1616-1683) très grand prédicateur calviniste.

11. Clarendon Code : il comprend les 4 statuts passés en 1661 et 1665.

12. Test Act : en 1673 puis en 1678 , cette loi impose à tous les non-anglicans, catholiques comme dissenters, de recevoir la communion selon le rite anglican et de répudier la transsubstantiation. 13. William Sancroft, 1617-1693. Doyen de St Paul en 1664, contribue à sa reconstruction après le Grand Incendie de Londres. Nommé archevêque de Canterbury en 1677, il préside au couronnement de Jacques II mais s'oppose à lui sur la Déclaration d'Indulgence et est emprisonné. Il s'opposera également à Guillaume d'Orange et sera suspendu de ses fonctions en 1690, pour être remplacé par Tillotson.

14. Pour davantage de détails on peut se référer à Thomas MACAULAY, History of England, London, 1848, encore considéré aujourd'hui comme digne d'intérêt pour sa recherche approfondie de la période, malgré son parti-pris whig.

15. Mort en 1688, fils du grand Increase Mather et frère de Cotton Mather.

\section{RÉSUMÉS}

Faut-il être doublement minoritaire en Angleterre à la fin du XVII ${ }^{\mathrm{e}}$ siècle pour mieux apprécier les vertus de l'adage: l'union fait la force? Il semble que ce soit le cas d'un prédicateur presqu'oublié de nos jours, Matthew Mead, né vers 1630 et mort en 1699. Appartenant au mouvement dissident fortement opposé aux Anglicans mais minoritaire après la mort de Cromwell, Matthew Mead vit les années tumultueuses de la Restauration et du retour de la monarchie Stuart au pouvoir, avant que la Glorious Revolution ne la chasse. Conscient de la faiblesse politique des «Independents » (congrégationalistes) dont il est l'une des grandes voix, il ne cesse de prêcher en faveur de "the happy union", mouvement de réconciliations entre presbytériens et congrégationalistes. L'échec de ses espoirs n'atténue en rien sa popularité, qui se manifeste en particulier lors de ses funérailles où les adversaires se retrouvent dans un même chagrin. 
At a time in England when religion cannot be dissociated from politics, Independent minister Matthew Mead (c.1630-1699) is nowadays almost forgotten, contrary to Baxter, another great voice among dissenters. Yet, he knew that all dissenters had to unite if they wanted to influence the leading politico-religious streams after the eviction of the Stuart monarchy following the Glorious Revolution. Hence his efforts, mostly through his preaching, to persuade both Congregationalists and Presbyterians to join "the happy union". His failure does not put an end to his renown, as shown by the crowds who met at his funeral service.

\section{AUTEUR}

\section{ARLETTE SANCERY}

Arlette SANCERY, agrégée d'anglais en 1961, exerce d'abord en lycée puis comme Assistante à l'université de Nanterre. En 1972, elle est nommée Maître Assistante à Paris XIII Villetaneuse où elle rencontre Lucienne Germain, alors jeune lectrice. C'est le début d'une amitié qui durera jusqu'au décès de Lucienne. Après plus de 22 ans passés à Villetaneuse, où elle est successivement chef du département d'anglais, assesseur auprès du directeur de l'UFR, présidente de la commission de la pédagogie de l'Université, Vice-présidente auprès du Président Cornillot et membre de la commission des relations internationales de Paris XIII, A. SANCERY obtient sa mutation à l'Université Paris IV-Sorbonne. Elle y finira sa carrière à l'UFR d'anglais tout en exerçant des responsabilités au sein du Bureau des relations internationales sous la présidence de JR Pitte.

Formée à la phonétique anglaise sous la direction du professeur Gimson à University College, Londres, son domaine de prédilection reste la linguistique historique vers laquelle MargueriteMarie Dubois, puis André Crépin de l'Université Paris IV la guident. L'histoire des mouvements religieux et l'évolution de la pensée religieuse en Angleterre du Moyen Age à la Réforme forme son $3^{\mathrm{e}}$ axe de recherche. L'article présenté aujourd'hui en hommage à Lucienne Germain se situe dans ce cadre. 